\title{
La búsqueda de los desaparecidos y los procesos de la identificación: una aproximación desde la antropología forense
}

\author{
María Inés Barreto R.*
}

\begin{abstract}
Resumen
La antropología forense en el Perú tiene un desarrollo reciente, ligado especialmente a las investigaciones que se efectúan en torno al Conflicto Armado Interno 1980 2000. Una de las líneas principales en dichas tareas se encuentra en la búsqueda de las personas desaparecidas y en la identificación de las víctimas exhumadas, muchas de las cuales se encuentran sin identificar. El documento busca presentar cada uno de los métodos de identificación existentes y su aplicación, su validez y aceptación en procesos judiciales, enfatizando en la importancia de acudir a métodos fehacientes asegurando un mínimo de confianza para todos los involucrados. Sentido se intenta explicar cómo y por qué es necesario valorar objetivamente cada método, tratando de reconocer con claridad el aporte y el papel que desempeñan en la identificación de víctimas del conflicto la antropología forense, los análisis osteopatológicos, los registros dentales y los análisis genéticos, teniendo en cuenta que sólo en la medida en que se disponga de una cantidad suficiente de elementos, se logrará una identificación fehaciente positiva o negativa-y se cumplirá el objetivo jurídico, social y psicoafectivo que se busca.
\end{abstract}

Palabras Clave

Antropología forense, identificación de personas, métodos de identificación, reconstrucción facial, superposición cráneo foto.

\begin{abstract}
The forensic anthropology in Perú has a recent development, because of the investigations assumed around the Armed Conflict Internal 1980-2000. One of the main issues in such tasks is the search of the missing persons and the identification of the victims exhumed, many of them remaining unidentified. The document pretends to illustrate about each one of the methods used for identification and its application, as well as its validity and its acceptance inside the legal processes, emphasising on the need of use reliable systems, to assurance a minimum of confidence to all those who are involved. The text tries to be exhaustive regarding to the systems of the facial reconstruction and the skull/photo superimposition, due to the over estimation of its effectiveness as reliable identification methods, during a considerable time. In that sense, the article tries to explain how and why it is necessary to value each method from an objective perspective, trying to recognise clearly the contribution and role of the forensic anthropology, the osteopathological analysis, the dental records, and the genetics, in the identification of victims of the Armed Conflict Internal. It is necessary to have in consideration that only by counting with a considerable amount of elements, a reliable identification positive or negative- will be possible, accomplishing the searched legal, social, psychological and affective purposes.
\end{abstract}

Keywords

Forensic anthropology, human identification, identification systems, facial recostruction, skullphoto superimposition.

\footnotetext{
* Docente Departamento Académico de Antropología y Arqueología UNMSM; Coordinadora Diplomatura en Antropología Forense y Derechos Humanos, Investigadora Principal CENIA.Email: mbarretor@unmsm.edu.pe
} 


\section{Introducción}

La antropología forense, sub-disciplina derivada de la bioantropología, surge recientemente en el Perú a partir de las investigaciones que se vienen realizando como consecuencia del Conflicto Armado Interno padecido entre 1980 y 2000, que dejó un altísimo número de desaparecidos más de 12000 personas'. Uno de los puntos trascendentales en las investigaciones que se efectúan actualmente, es la identificación de las víctimas exhumadas, muchas de las cuales se encuentran en condición de $\mathrm{Nn}^{2}$.

Desde hace largo tiempo se ha buscado lograr la identificación fehaciente en términos biológicos, jurídicos y sociales- de los individuos desde diferentes perspectivas. Mediante múltiples investigaciones, la mayoría centradas en el campo de la biología humana y, partiendo del hecho de que más que una necesidad es un derecho y un principio reconocer y ser reconocidos legal y socialmente, la comunidad científica ha tratado de encontrar el método para identificar positivamente a personas -vivas o muertas- no identificadas.

Aún cuando en el transcurso de los dos últimos siglos se han obtenido enormes avances en éste terreno, su aplicación en la búsqueda de personas desaparecidas como consecuencia de conflictos armados o de situaciones de alteración del orden público, entre otros, ha obligado a la búsqueda de métodos efectivos, en especial cuando se trata de restos esqueletizados o en proceso de esqueletización, donde se han perdido la mayor parte de posibilidades de que los cuerpos de las víctimas logren ser restituidos a sus familias y comunidades.

Algunos investigadores han aportado desde la antropología forense desconociendo que sus principales objetivos, contribuir en la identificación de personas desaparecidas y contribuir a establecer las causas de la muerte, requieren de un abordaje multidisciplinario e integral que permita llegar a conclusiones serias.

En el proceso de identificación, que es el que nos interesa abordar en el presente texto, se han aplicado diferentes métodos y técnicas que van desde el reconocimiento visual del cuerpo o de las prendas, pasando por la descripción de las características morfológicas (o descripción física) el análisis de la dentadura, hasta las más modernas y recientes técnicas de filiación a partir del ADN. Su correcta utilización debería servir a todos aquellos interesados en la identificación de víctimas, es decir, familiares, autoridades y acusados; sin embargo, el desconocimiento, así como la sobre dimensión de algunas de las técnicas pueden conllevar a que muchas de las víctimas permanezcan sin identificar o se les asigne una identidad equivocada, con las graves consecuencias que cualquiera de estas situaciones pueden provocar. El fin de este texto, por tanto, no sólo es informar sino invocar a aquellos interesados y especialistas a acudir a los métodos y técnicas adecuados para garantizar que al menos uno de los objetivos del proceso de autopsia, en el que la antropología forense contribuye, se lleve a cabo exitosamente.

\section{Breve historia de la identificación de perso- nas}

La identidad es aquello que permite a los individuos reconocer y ser reconocidos como parte de un grupo familiar y social. Además determina su existencia jurídica y los hace acree papel fundamental en las sociedades, que han creado diversas formas para lograr caracterizar a cada uno de

\footnotetext{
1 Uno de los productos dejados por la CVR es la lista Los Peruanos que Faltan, donde figuran reportados 8558 desaparecidos. A ésta se sumaron casos nuevos aportados como parte de la campaña Construyendo una Esperanza: Los Desaparecidos en el Perú, de la Coordinadora Nacional de Derechos Humanos, con lo que la cifra total de personas desaparecidas llega a ser de 12027 a nivel nacional, a la fecha.

2 El término NN se refiere a Ningún Nombre y proviene del latín Non Nomine, sin nombre conocido.
} 
sus miembros, mediante prácticas y rituales que permiten la aceptación de un individuo como parte de un grupo específico.

En las ciencias forenses, lograr la identificación de un individuo, un objeto una situación, ha llevado a que se profundice en la búsqueda de métodos que permitan establecer de manera precisa tal identidad. Así, se ha procurado diseñar formas eficientes para definir con certeza la pertenencia de un objeto o sujeto "X" a un entorno determinado o la uniprocedencia del mismo.

Lograr la identificación de personas en un universo dado tiene sus bases en elementos biológicos, pero estas no pueden ser vistas aisladas del carácter social y jurídico que se encuentran implícitos en dicho proceso; y que buscan en conjunto, fijar con precisión y sin lugar a equívocos el reconocimiento de una persona, viva o muerta, de manera que al hacerlo su identidad no sea fácilmente modificable y la persona logre ser reconocida cada vez que se requiera.

Es muy posible que los primeros métodos de identificación se limitaran a establecer el nombre con base en algunas señas particulares como la descripción de oficios o determinadas características de las personas requeridas. Sólo en la medida en que las sociedades se complejizan, se empiezan a buscar métodos de identificación más exactos. Un claro ejemplo de ello lo constituye la existencia de documentos de identidad, práctica generalizada en las sociedades actuales, que permi-

\footnotetext{
Figura 1. Foto del Museo de la Prefectura de París, donde se muestra una tarjeta de identificación señalando las características antropométricas de

la persona, su foto y sus huellas dactilares (Tomado de $h t t p: / / w w w . c i t e-c i e n c e s . f r / f r a n c a i s /$
}

te la vinculación de las personas a un sistema social específico y les garantiza entre otros aspectos, su reconocimiento legal.

Sin embargo, mientras se universalizó la utilización de un documento de identidad y dada la necesidad de contar con un instrumento de identificación legal, en algunos países europeos se recurrió a la descripción de las características morfológicas y de los rasgos faciales y corporales, mediante la antropometría o "medición de cuerpos”, principalmente con fines legales y penales. Este sistema, bastante popular en el siglo XIX, fue materializado por el francés Alphonse Bertillon, que en 1880 crea el concepto de "retrato hablado", el cual permitía conocer las dimensiones corporales del cuerpo humano en individuos adultos con fines de identificación personal siendo su uso predominante durante un período considerable, por lo que también se le llamó "Bertillonaje" (Fig. 1). Se basaba en la idea de que existían características individuales únicas fenotípicas- que permitirían el reconoci-

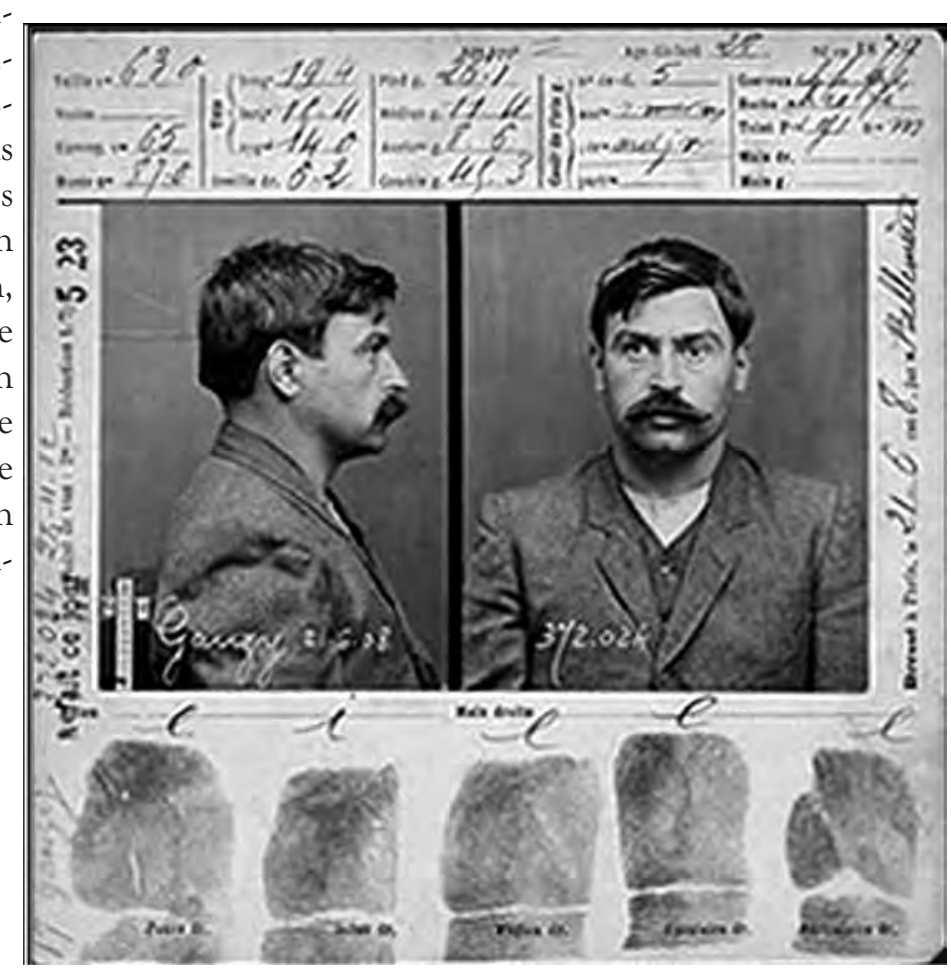


miento total que tan necesario se hacía y se constituyó en el mayor aporte a la identificación de personas, por cuanto tomaba como base el estudio de las huellas plantares, palmares y dactilares, así como también las dimensiones de órganos como las orejas.

El uso de la antropometría aplicado a la criminalística, pretendió por un tiempo fijar con precisión la identidad de los delincuentes. De la aplicación de ésta técnica surgieron propuestas como la de César Lombroso, representante del positivismo criminológico italiano, que planteaba en 1876 en una de sus primeras obras ${ }^{3}$, la posibilidad de caracterizar al "criminal nato". Según esta escuela, conocida también como la de la Antropología Criminal, a la que se unieron entre otros, personajes como Enrico Ferri, ciertas características físicas estarían vinculadas a la comisión de determinados delitos; de manera tal que para detectar a los delincuentes, se recurría a la medición y descripción morfológica, las que, en conjunto, permitirían establecer que quien se ajustara a determinado perfil, ya preestablecido, podría ser juzgado por su apariencia y sus rasgos físicos y no necesariamente por la comisión real de un delito.

Por razones de corte discriminatorio, pero además por no ser lo suficientemente precisos, los métodos derivados de la escuela Lombrosiana no se expandieron; al menos no lo suficiente, a pesar de que buena parte de nuestra sociedad esta viciada por estos conceptos y los juicios basados en el aspecto físico sigue siendo un elemento que prima en las relaciones sociales.

Sin embargo las investigaciones continuaron y así el estudio del iris de los ojos, de las orejas, del ombligo, entre muchos otros, guiaron al hallazgo de métodos más precisos, económicos y fácilmente aplicables (Sánchez y Vargas, 1993;
Lorente, 1995). Además se llevan a cabo los primeros estudios sobre reconstrucción facial, aplicados sobre todo a casos históricos (Krogman e Yscan, 1986).

La dactiloscopia, uno de los métodos más populares, tuvo sus inicios a mediados del siglo XIX, a partir de los trabajos del inglés Francis Galton. Sin embargo, uno de sus principales exponentes es el argentino Juan Vucetich, quien a principios del siglo XX creó el Sistema Dactiloscópico Argentino, posteriormente aplicado en varios países hasta la aparición de nuevos sistemas más modernos. En este mismo período se incorpora el análisis de la dentadura con fines de identificación forense (Valdés, 1995), que aún muestra resultados positivos.

Es recién en la década de 1980, cuando comienzan a ser aplicados en Inglaterra por A. Jeffreys, los estudios sobre la descendencia y la transmisión de caracteres planteados por Mendel y desarrollados posteriormente por otros investigadores en casos forenses de manera exitosa (Lorente, 1995). Todos estos estudios mencionados constituyen la base actual para la identificación de personas en ciencias forenses.

\section{Principios para la identificación}

El objeto central de la secuencia de estudios descrita es seguir el principio de identidad según el cual “...un pensamiento o un objeto es idéntico a si mismo siempre que sus rasgos no varíen en el tiempo a pesar de las circunstancias a que sea sometido. Esta ley puede resumirse en la fórmula $A$ es $A$, donde A es un objeto, evento, proceso, acción o cualidad cualquiera" (De Gortari Eli, 1972 en Sánchez y Vargas, 1993:8).

Se debe tener en cuenta que el ser humano es producto de la variabilidad filogenética, ontogénica y sexual que lo hace individualmente

\footnotetext{
3 L'uomo delinquente. 1876. Su 5ª edición consta de tres tomos: I.- L'Uomo, II.- Crimen, causas y remedios, III.Atlas.
} 
único y diferente pero a la vez le otorga características comunes a toda la especie. Desde este punto de vista, lo que se pretende es establecer aquello que es común a todos y diferenciar lo que es propio y único en cada uno.

Existe variabilidad a nivel sexual. Hombres y mujeres guardan características biológicas que los particularizan y asignan dentro de uno de los dos sexos, con algunas excepciones de índole genética. Así mismo, se conservan algunos rasgos filogenéticos propios de la especie humana que también aparecen en otras especies, dada nuestra condición de mamíferos y vertebrados. Cabe recordar que los seres humanos compartimos con nuestros primos más cercanos, los chimpancés, casi un 99\% del material genético. Sin embargo, somos tan diferentes de ellos que hacemos parte de especies distintas. El ser humano ontogénicamente cambia desde la concepción hasta después de la muerte a pesar de lo cual, hay características inherentes a cada uno que no van a variar sustancialmente con la edad.

Es justamente con base en la variabilidad, que se debe buscar aquello que hace a un individuo completamente diferente de los demás y que se mantendrá inalterable a pesar de las circunstancias. Lo que se requiere entonces para determinar la identidad es que la esencia física del objeto en cuestión se conserve rigurosamente inmutable, a pesar de los cambios originados por causas naturales, artificiales o accidentales.

En relación con los procesos legales, se requiere que el principio de identidad mantenga su valor diferenciador, donde cada elemento y, para el caso que nos atañe, cada individuo, será único, diferente a los demás e idéntico a si mismo y para lograrlo este ser debe distinguirse de los otros. La distinción se basará en una serie de signos externos y/o internos, según sea el caso, que permitirán reconocerlo e identificarlo como tal.

En ese mismo orden y teniendo como premisa la responsabilidad social además de la legal, es importante que la identificación se realice de manera que no deje lugar a dudas. Tanto en casos de personas acusadas de delitos, como en casos de personas cuyo paradero se desconoce, es necesario que se acuda a métodos fehacientes, es decir indiscutibles e indubitables, donde las características en las que se basen las conclusiones para establecer la identidad sean tan precisas como sea posible.

Para lograr la identificación de las personas se acude a métodos que permitan fijar sus características de manera cierta, objetiva, durable, invariablemente reconocible y fácilmente observable. Lo anterior en tanto la identidad es un hecho, además de ser una convención, más no es un criterio. A través de ella la sociedad, la ley y los estados buscan un medio eficaz y seguro para garantizar el reconocimiento cultural, pero también el orden social, así como la defensa de la individualidad y de la colectividad (Sánchez y Vargas, 1993).

\section{Bases del proceso de identificación}

Para lograr una identificación adecuada se deben seguir una serie de pasos básicos, aplicables no sólo en el campo forense sino en cualquiera que requiera establecer la misma procedencia o uniprocedencia de un elemento o un hecho.

Durante el análisis de restos de personas fallecidas que permanecen sin identificar (esto es como NN), el objeto es llegar a establecer una identidad con base en un reporte previo de desaparición, que será comparado con el resultado del análisis de los restos en cuestión. Retomando la propuesta de Sánchez y Vargas (1993) se aplicarían los criterios que se describen a continuación a lo largo de todo el proceso:

En primer lugar se tiene la observación como punto de partida. Se lleva a cabo mediante la exploración, buscando todas las señales que particularicen a un sujeto determinado. Debe ser objetiva y en esta medida selectiva, pues sólo se van a registrar los elementos característicos e 
individualizantes. La observación deberá ser interpretativa, conllevando a una conclusión.

A la observación le sigue la descripción, que debe ser lo más detallada posible, realizándose con base en la observación. En esta etapa de trabajo se debe resaltar lo más característico del objeto analizado.

El paso final es la confrontación, entendida como la comparación de descripciones, no de objetos, sujetos o fenómenos, ya que estos tres existen de manera indubitada y son los patrones de comparación. Son sus características las que han de confrontarse y establecer o no una similitud y con base en ella una identidad.

Estos pasos, en los procesos forenses estarán a cargo del perito, quien en virtud de su experiencia y conocimientos amplios y específicos en un campo, disciplina o profesión determinada, observará, describirá y comparará; ofreciendo un resultado a la autoridad competente, quien a su vez, basado en el criterio del experto deberá llegar a una conclusión.

Un aspecto básico a tener en cuenta para la valoración de pruebas y específicamente para la identificación de personas, es la cantidad y calidad de elementos que permitirán llegar a una conclusión determinada. $\mathrm{Al}$ respecto debe tenerse en cuenta la ley del cálculo probabilístico, propuesta por Velásquez, L.G. (1990, en Sánchez y Vargas, 1993) que establece que " $a$ mayor cantidad de características concurrentes entre los elementos cotejados mayor probabilidad de uniprocedencia". Como esta es una ley de orden cuantitativo, es necesario tener en cuenta además, las particularidades cualitativas, las características que por si solas no permiten identificar pero tienen un gran valor dentro de los porcentajes de probabilidad, en donde la posibilidad de que estas se repitan en una población son pocas o inexistentes.

Siguiendo los pasos descritos y teniendo en cuenta la ley de cálculo probabilístico, el perito deberá presentar su dictamen, estableciendo la probabilidad de que las características observadas, descritas y confrontadas se repitan en la población y por tanto sea posible disponer de los elementos de juicio suficientes para lograr una identificación fehaciente.

Como resultado de esta secuencia se obtiene el juicio de identidad. Por lo general el juicio está conformado por la conclusión del perito y la decisión de la autoridad competente. Será ésta última quien decida y logre establecer, teniendo en cuenta la evidencia presentada por el primero, si se ha llegado a una identificación fehaciente (CVR, 2003).

En ese sentido, la identificación ofrece dos opciones básicas: positiva o negativa. Una identificación será fehaciente positiva, en la medida en que las características concurrentes sean tantas y tales que permitan llegar a un juicio de uniprocedencia. Mientras tanto, será fehaciente negativa, descartando la identificación, si las características comunes no son suficientes o son irrelevantes. Pueden haber algunas categorías, sin embargo, que se presentan, cuando la identidad es indiciaria o tentativa, es decir, cuando se tienen algunos elementos que permiten pensar que hay uniprocedencia, pero son insuficientes para afirmarlo de manera categórica.

Hasta aquí se ha comentado acerca de la identificación y la individualización, como dos puntos básicos que pueden estar relacionados, formando parte de un mismo proceso, en la medida en que para que haya identificación ha habido un proceso previo de individualización, es decir de particularización. Llegar a esta última, sin embargo, no garantiza la identificación plena de un individuo.

Según el diccionario de la Real Academia de la Lengua Española se define Identificar como "...reconocer si una persona es la misma que se supone o se busca", es decir, comprobar que dicha persona es la misma conocida en otras circunstancias o de la que se poseen ciertos datos "... parte del concepto de persona como diferente al resto de los 


\section{Métodos de identificación}

Cuando se hace el hallazgo de cadáveres cuya identidad se desconoce, uno de los pasos primordiales a seguir dentro del proceso es lograr establecer con precisión quiénes eran las víctimas y cuáles fueron las causas de su muerte. En el Perú como consecuencia del período de Conflicto Armado Interno padecido entre los años 1980-2000, este es un requerimiento necesario que obliga a agotar los métodos investigativos para reducir la impunidad reinante y lograr identificar a víctimas y victimarios relacionados en causas legales. Esto aplica en especial dentro del ámbito de los derechos humanos, sin embargo no se deben dejar de lado las miles de muertes que se producen anualmente por causas de delincuencia común que ameritan métodos igualmente efectivos para la identificación de víctimas. Dentro de los métodos fehacientes está el análisis de las huellas digitales, el cotejo odontográfico, la comparación de historias clínicas, así como el análisis de ADN. Todos sin excepción requieren de información previa con la cual comparar.

El primer método al que se recurre por lo general, es a la comparación de las huellas dactilares con los registros disponibles en archivos oficiales. Cuando es imposible recuperar las huellas o no existen registros previos, se debe recurrir a otros sistemas. Los países latinoamericanos son de los pocos en donde se toman huellas digitales como parte del proceso para obtener el documento de identidad. No obstante no toda la población hace parte del sistema de identificación, establecido básicamente con fines electorales, a lo cual se suma el que la población más afectada por la violencia, es la que se encuentra en las zonas más alejadas, más pobres y con menos posibilidades de acceder siquiera al documento de identidad.

En otros países las huellas digitales se toman sólo en casos de competencia legal, a delincuentes o sospechosos de delitos. Sin embargo, cuen tan con un sistema de salud con amplia cobertura, que permite el acceso de la mayor parte de la población a los servicios de salud general y oral y por ende a un registro clínico y odontológico actualizado. Sin entrar en detalles, cuando no se cuenta con la huella dactilar, otros métodos de identificación a los que se acude son el cotejo de odontogramas (odontográfico) y a las historias clínicas. Estos, al igual que la huella dactilar permitirán en una cantidad considerable de casos identificar fehacientemente a los individuos que asílo requieran. Al último método al que se recurre para la identificación de víctimas $\mathrm{NN}$, es el análisis del ADN, sea nuclear, mitocondrial o cromosómico.

\section{La dactiloscopia}

Las huellas dactilares constituyen una de las pruebas físicas más comunes y de mayor valor para la identificación de personas y, desde 1891, son utilizados en diferentes lugares del mundo con este fin. Su valor se encuentra en que es un elemento universal pues todo ser humano las tiene. Además y dentro de este elemento universal, existen tres características que son fundamentales en su uso y que las hacen decisivas a la hora de establecer o verificar individualidades (Lubian y Arias, 1975).

La primera característica es que las huellas dactilares son perennes. Aparecen a partir del $4^{\mathrm{O}}$ mes de vida intrauterino aproximadamente y permanecen durante toda la vida, hasta avanzados estados de descomposición, incluso por cientos o miles de años en casos de estados conservativos de la descomposición, como la momificación.

La segunda característica es que son diversiformes. Presentan una variedad infinita, de modo que son únicas y al parecer no existen dos personas con el juego de huellas digitales idéntico, aún en casos de gemelos monocigotos, cuyos dibujos son idénticos pero en sentido inverso, es decir en la mano contraria (Simonin 1982). En conse- 


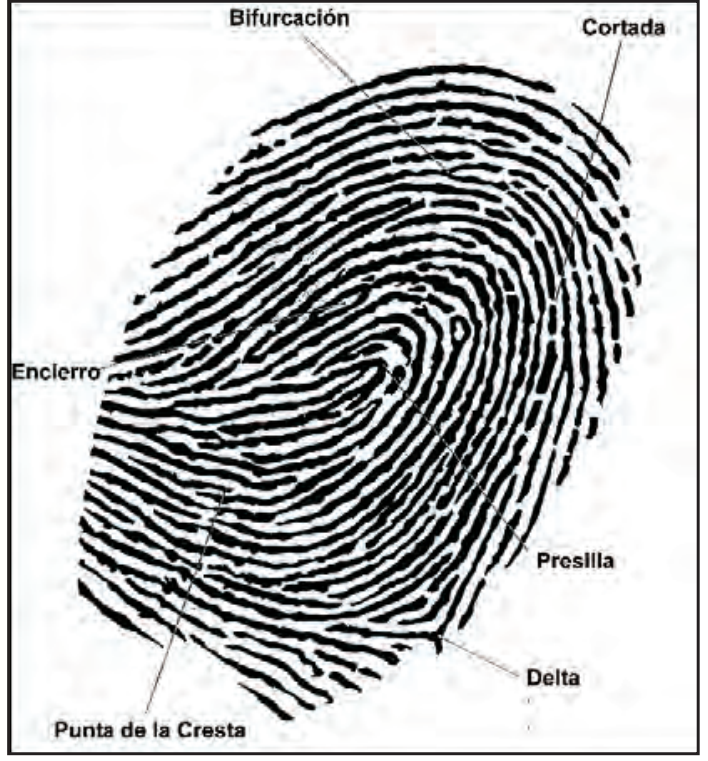

Figura 2. Las huellas digitales presentan características únicas e irrepetibles. En el gráfico se observa la representación de algunas de ellas (Modificado de http://www.barcode.ro/ Tutorials/biometrics/fingerprint.html).

cuencia, presentan una variedad, individualidad y especificidad que las hace únicas en cada persona.

La tercera característica es que son Inmutables. Esto es, son inalterables, bajo ninguna circunstancia pueden modificarse, a menos que se afecten las capas más profundas de la piel, de lo contrario el tejido se regenera manteniendo la forma de las huellas intacta.

Para complementar esta serie de ventajas, la información obtenible de las huellas se puede clasificar y sistematizar fácilmente, estableciendo por lo menos nueve puntos de coincidencia (desde un solo dedo hasta las dos manos) que al momento de realizar la comparación permiten concluir fehacientemente con la identidad del individuo cuestionado (Foto 2). Sin embargo en casos donde la putrefacción está muy avanzada o el tejido blando ha desaparecido o cuando no se dispone de elementos de cotejo suficientes, se debe recurrir a otros métodos.

\section{El odontograma (Carta dental)}

El odontograma, basado en la odontoscopia, se desarrolla considerando que los dientes van a ofrecer características similares a las que presentan las huellas dactilares, aunque con muchos más inconvenientes que no han sido aún resueltos como se describe más adelante.

El análisis parte de considerar que los dientes son la estructura más dura del cuerpo humano, resistiendo temperaturas mayores a los 1000 grados centígrados. El número de dientes que poseemos por lo regular los seres humanos, es de veintiocho a treinta y dos dientes en adultos, donde la fórmula dental es de 2123, esto es 2 incisivos, 1 canino, 2 premolares y tres molares en cada hemimaxila. A esto se le debe agregar que cada uno de los dientes presenta 5 caras, oclusal, medial, distal, vestibular (o bucal) y lingual (o palatina), con características genéticas particulares y cuyas transformaciones dadas a lo largo de la existencia de la persona, no se repetirán estadísticamente sino con una frecuencia mínima.

En estos análisis, además del número y características de los dientes, se debe tener en cuenta todo el sistema estomatognático, es decir las mucosas, los músculos y otros elementos relacionados con la masticación e incluso en parte, con la digestión (Caballero 2006) .

Los inconvenientes sin embargo, se relacionan con las condiciones necesarias para lograr una identificación fehaciente. En primer lugar, no hay consenso alrededor de la cantidad de características mínimas requeridas cuando de comparar se trata. Todo dependerá de la calidad de las pruebas y la posibilidad de que estas se repitan poblacionalmente, variando para cada caso en particular.

En segundo lugar, una de las fallas más grandes que se observa, al menos para el caso latinoamericano, en donde los sistemas de salud no tienen una cobertura lo suficientemente amplia, es justamente que no todas las personas tiene acceso a la atención odontológica y cuando se tiene 
esa posibilidad, no todos los profesionales en el área de la odontología realizan un odontograma físico con la información de cada paciente. A lo anterior cabe añadir que esta información puede variar drásticamente en lapsos cortos, razón por la cual el método puede no ser tan útil cuando se requiere (Fig. 3). Sin embargo cada caso es diferente y este podría convertirse en uno de los mejores sistemas de identificación, en la medida en que se profundice en la investigación en este campo, en especial en casos donde los restos se encuentran esqueletizados o en estados avanzados de descomposición, por cuanto es un método económico y a pesar de sus carencias, fiable.

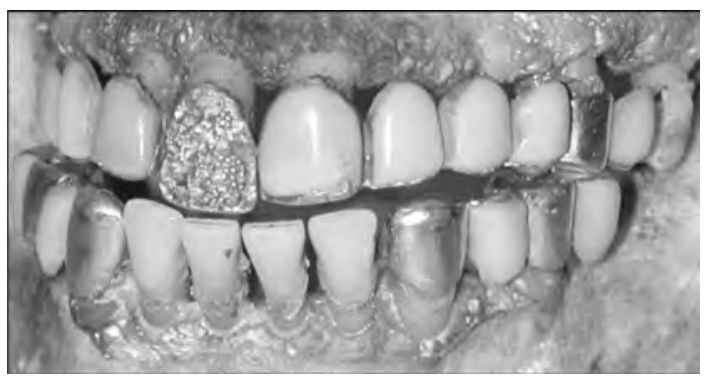

Figura 3. Los dientes, en especial cuando existe tratamiento odontológico regular o cosmético y su registro odontográfico e incluso testimonial, permite lograr la identificación fehaciente de la persona buscada (Foto: archivo personal).

\section{El ácido desoxirribonucléico (ADN)}

Después de los métodos mencionados, la utilización del ADN en casos forenses es uno de los que mejores resultados ha presentado en los últimos años. Los primeros análisis llevados a cabo en este campo estaban referidos a la toxicología y al estudio de algunos restos biológicos. Sin embargo por las limitaciones técnicas del momento, no fueron útiles como medios de identificación fehaciente en muchos casos (Lorente, 1995).

Esta técnica parte de varias premisas que permiten su utilización frecuente con resultados positivos en casos de identificación de personas cuando no se cuenta con mayores posibilidades.
El ADN es una sustancia que se encuentra presente en todas las células del cuerpo humano, siendo único e irrepetible en cada individuo ya que es polimórfico, por lo que permite, al ser analizado con las técnicas y bajo los parámetros adecuados, llevar a cabo análisis de filiación y/o identificación que presentan una alta fiabilidad.

Se pueden obtener resultados de cuerpos de personas después de cientos y en ocasiones de miles de años, dependiendo de que los procesos tafonómicos y químicos relacionados tanto con el individuo, así como los relativos al mecanismo que produjo la muerte y el medio en que la descomposición se dio, no afecten su estructura. El análisis del ADN permite comparar los restos (hueso, sangre, músculo, pulpa dental) de la víctima con restos de la misma que se hayan mantenido con anterioridad a su desaparición (p.e. dientes, cabello, sangre, entre otros); o con muestras de los familiares (sangre, saliva) lo cual permite establecer vínculos de parentesco biológico, hasta lograr una aproximación lo suficientemente certera, incluyendo o excluyendo a una persona en un proceso de identificación (Lorente y Lorente, 1995).

Los requisitos para llegar a una conclusión efectiva están basados tanto en la calidad como en la conservación de la muestra es decir que se pueda obtener material genético relevante de la misma. En ese sentido, se debe tener en cuenta que no todas las muestras permiten extraer ADN. No es igual una muestra de sangre reciente, a un fragmento de hueso que haya estado sometido a altas temperaturas o a factores externos que hayan podido afectar la calidad del material genético. Así mismo, se requiere contar con elementos de cotejo, sean de la víctima o familiares cercanos, sin los cuales la obtención de cualquier muestra es poco útil (Fig. 4) .

A lo anterior debe agregarse que el laboratorio que lleve a cabo el examen debe disponer del equipo tanto técnico como científico adecuado, para garantizar que las pruebas serán procesadas- 


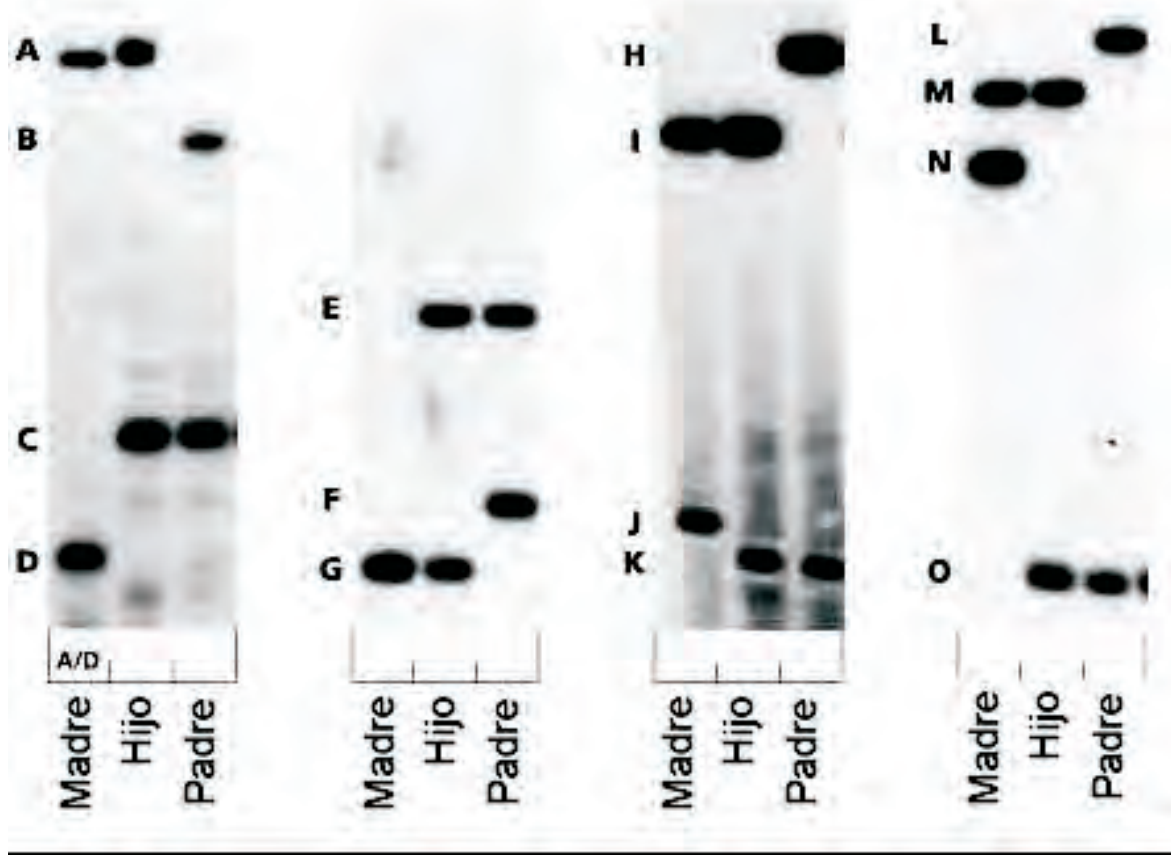

Figura 4. Comparación de perfiles genéticos. Nótese que padres e hijos comparten al menos un elemento en común (Modificado de http://wwww.pbs.org/wgbh/aso/resources/guide/images/dnafingerprint.jpeg).

de manera efectiva, emitiendo resultados cotejables y aptos que permitan emitir juicios de identidad certeros e invariables. Es importante que la muestra poblacional con la que se trabaje esté adaptada a la región o país sobre la que se ha de aplicar para lograr porcentajes reales y altos de coincidencia. De la misma manera el laboratorio debe contar con las certificaciones y convalidaciones necesarias, ya que no cualquier laboratorio de genética se encuentra en capacidad de responder a la realización de análisis en restos humanos esqueletizados o en descomposición y por demás no todos los laboratorios se encuentran certificados mundialmente para ésta tarea, al menos en el área forense.

Hasta la fecha, el análisis del ADN sea nuclear, cromosómico o mitocondrial, puede ser utilizado como paso al cual acudir cuando ninguna otra técnica de identificación es aplicable, logrando en la mayoría de casos una identificación fehaciente. Uno de los inconvenientes que presenta es que el resultado ofrece una filiación familiar, más no una identificación plena del individuo porque pocas veces se cuenta con información genética previa. Por demás sobra resaltar que se trata de análisis costosos y que deben ser realizados por laboratorios con personal y equipos especializados. A esto se añade que cuando el material genético no se ha conservado adecuadamente, se corre el riesgo de no llegar a obtener y/o procesar las muestras y por ende no lograr llegar a conclusión alguna. Por tales razones se recomienda acudir a él una vez se hayan agotado todos los demás recursos existentes.

Los métodos descritos son los básicos a la hora de identificar restos de individuos que se encuentran en calidad de NN. Se puede acudir a métodos complementarios e igualmente válidos, como por ejemplo el cotejo de lesiones osteopatológicas. Esto es, las lesiones que dejan huella en el hueso como consecuencia de ciertas patologías. Esto puede llegar a ser de gran utilidad, en 


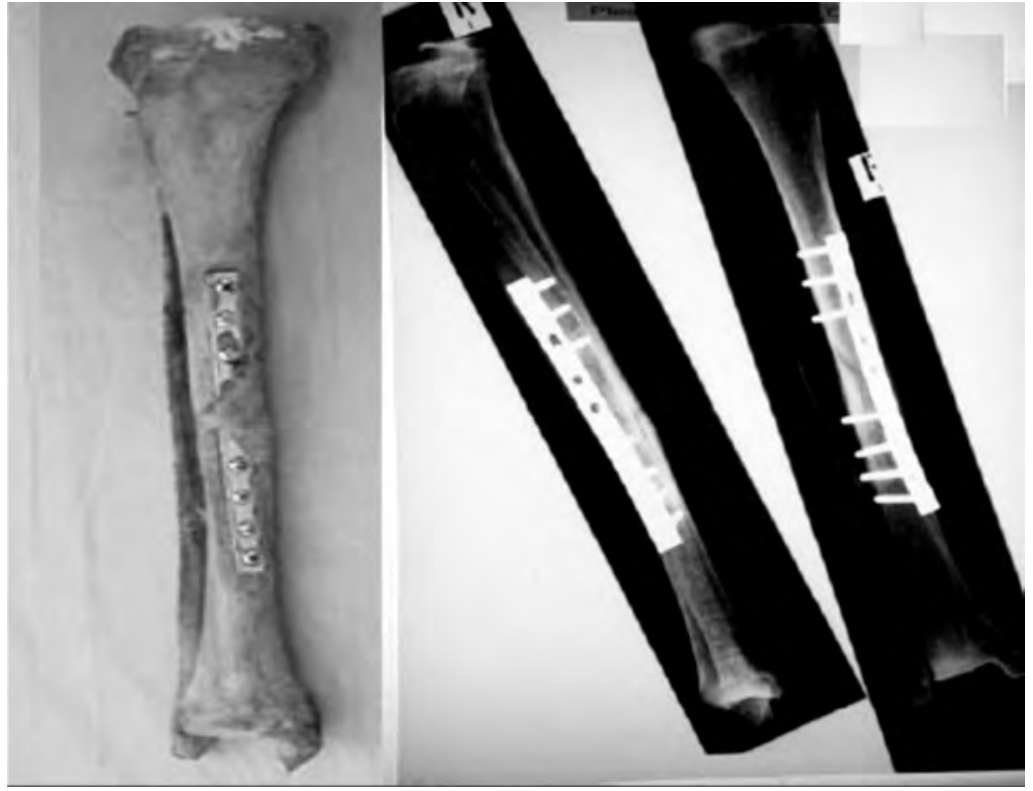

Figura 5. Algunas fracturas ocurridas en vida cuentan con tratamiento especializado e historia clínica con radiografías, lo que permite la identificación fehaciente de la víctima (Foto archivo personal).

la medida que se cuente con la información preliminar suficiente y adecuada para tal fin. El mejor ejemplo y el más sencillo se da en los casos donde existen fracturas donde la identificación podría ser fácilmente realizable de contar con la historia clínica o con un relato preciso del tipo de lesión. Si en una fractura se encuentran elementos de osteosíntesis, utilizados para su reducción en casos graves, la identificación será aún más rápida y eficaz siempre y cuando exista la documentación clínica que permita hacer una comparación entre lo observado y lo descrito (Fig. 5) .

Igualmente, si se logra cotejar de manera efectiva la descripción brindada por familiares referente a enfermedades sufridas por la víctima desaparecida ("cojera", "le dolía el cuello...", "le dolía la espalda", "tenía dolor de muela" "le faltaban dientes") con los hallazgos realizados en los restos analizados, en la medida en que la cantidad de elementos coincidentes sea suficiente para sustentar una identidad que en éste caso se dará por "preponderancia de evidencia", donde apesar de la inexistencia de registros clínicos u odontológicos hay información ofrecida por allegados (Burns, 1999).

\section{Antropología forense e identificación de NNs}

En este punto es necesario advertir que no en todos los casos que se analizan se requiere necesariamente realizar un proceso de identificación completo de las víctimas. Desde otra perspectiva, no todos los NN son absolutos, es decir, aquellas personas que desaparecen y de quienes no se vuelve a tener noticia o de quienes no se tiene ninguna información disponible. No son pocos los casos, en especial en el Perú debido a las características del conflicto, en que los familiares, allegados o conocidos saben o tienen noticia del paradero final de las víctimas. En muchas ocasiones son ellos quienes los han inhumado, bien porque presenciaron su muerte, porque fueron informados del destino final de los cuerpos, o bien porque los buscaron incansablemente hasta dar con los cuerpos. 
En estos casos por tanto, la identificación es un proceso relativamente menos complejo porque se conocen de manera previa los nombres de las víctimas, pasando a ser más una ratificación de identidad, que un proceso de identificación como tal. A lo anterior, se suman las características geográficas y las dificultades de comunicaciones en muchas comunidades, que hacen que en muchos casos nos encontremos frente a víctimas que pueden ser consideradas como NNs relativos. Es decir, personas cuyos cuerpos en algún momento aparecieron luego de los hechos que llevaron a su desaparición y fueron inhumados de nuevo, y/o donde las familias cuentan con información suficiente sobre ellos, por lo cual realizar análisis especializados va a ser dispendioso, costoso e inasequible, la mayor parte de las veces, dadas las condiciones de infraestructura nacional, donde los especialistas en análisis especializados son pocos, al igual que los recursos para llevar a cabo dichos análisis.

Cualquiera que sea la situación, pueden realizarse análisis útiles que permitan bien sea individualizar o identificar. De acuerdo nuevamente, al diccionario de la Real Academia, Individualizar quiere decir como caracterizar, particularizar, comunicar o atribuir a un ser características que lo distinguen de los demás de su especie. Cuando hablamos de individualizar partimos del hecho de que hay un individuo que forma parte de un grupo social, de una comunidad, que presenta una serie de características generales que lo distinguen de otros; pero al tiempo tiene otras características que lo integran a ese grupo. Algo tan general que no le permite ser identificado sólo por esas características, pero tan particular que permite vincularlo a un grupo determinado.

Este es uno de lo mayores aportes de la antropología forense en lo que a la identificación de personas se refiere. Por esta razón en el tema de la identificación desde ésta disciplina, se recurre a algunas de sus técnicas más reconocidas, entre las que se cuentan la reconstrucción facial y la superposición cráneo-foto, sin dejar de lado el reconocimiento de prendas así como la información brindada por familiares y allegados de víctimas. Todos estos son considerados métodos indiciarios o tentativos.

Cabe recordar que cuando se recurre a la antropología forense es porque usualmente los restos en cuestión se encuentran en estados avanzados de descomposición o esqueletizados. En ese sentido el rol del antropólogo forense en todo momento debe conducir a reducir el universo, es decir a lograr la individualización que culminará en una posterior identificación, además del ya reconocido aporte de material probatorio que pueda fortalecer los indicios de responsabilidad en casos de índole penal.

Por lo tanto, su propósito con las herramientas técnicas y metodológicas de que dispone, está dirigido a proveer los elementos necesarios y suficientes para individualizar, más no para identificar, debido a que la mayor parte de la información que se obtendrá de esta disciplina se basa en estimaciones. En consecuencia, muy pocas de las conclusiones estarán dadas por determinaciones que permitan establecer con precisión que la información que se consigna es invariable, pues es de carácter tan general, que no permitirá establecer una identificación fehaciente. Por esta razón, el trabajo interdisciplinario es necesario, pues se debe complementar con el estudio de las huellas dactilares y cuando esto no sea posible, que para lo que nos atañe es la mayor parte de las veces, se debe acudir al cotejo de posibles patologías, de la carta dental, del ADN y en general con todos aquellos rasgos y elementos que permitan la identificación fehaciente de un individuo. A mayor cantidad de características mayor probabilidad de uniprocedencia.

El primer paso a seguir en cualquier análisis de restos óseos se refiere a determinar la especie biológica a la que pertenecen los restos, como condición básica para cualquier estudio, es decir, 
si los restos son humanos o animales. Luego se procede a establecer el número mínimo de individuos, seguido de un análisis sobre cada uno de estos, determinando el sexo, estimando la edad y la estatura así como las características morfológicas básicas, que en algunos casos podrá conllevar a la reconstrucción facial o a la superposición cráneo foto en caso de contar con los elementos suficientes. Si los pasos anteriores no se han llevado a cabo de manera precisa, por una persona capacitada o un perito, el proceso de identificación puede no darse adecuadamente. El análisis de patologías tanto odontológicas como corporales es un estudio que debe realizarse de manera interdisciplinaria y conjunta con médicos y odontólogos para lograr observaciones, descripciones y confrontaciones precisas.

Los métodos a los que se recomienda acudir en menor medida en el análisis antropológico forense es a la reconstrucción facial o la superpo- sición cráneo foto, por cuanto son dispendiosos y no han probado su efectividad en ninguna latitud en donde han sido aplicados. La primera se recomienda sólo en aquellos en que no se cuenta con indicios que permitan sospechar la identidad de un individuo, para publicar una imagen que convoque a posibles familiares a acercarse a ofrecer datos; y la segunda cuando existen similitudes entre un $\mathrm{NN}$ y un desaparecido creándose la expectativa de una posible identificación. Sin embargo, estos métodos son tan inexactos como el reconocimiento del cadáver o de las prendas de vestir, por lo que no deben prevalecer como elemento de juicio para identificación.

Algunos forenses han planteado la posibilidad de lograr identificaciones con base en el reconocimiento por parte de los familiares de las prendas de vestir y los objetos personales, que eventualmente se encuentran asociadas a los cuerpos (Fig. 6). Este proceso además de doloroso, ha

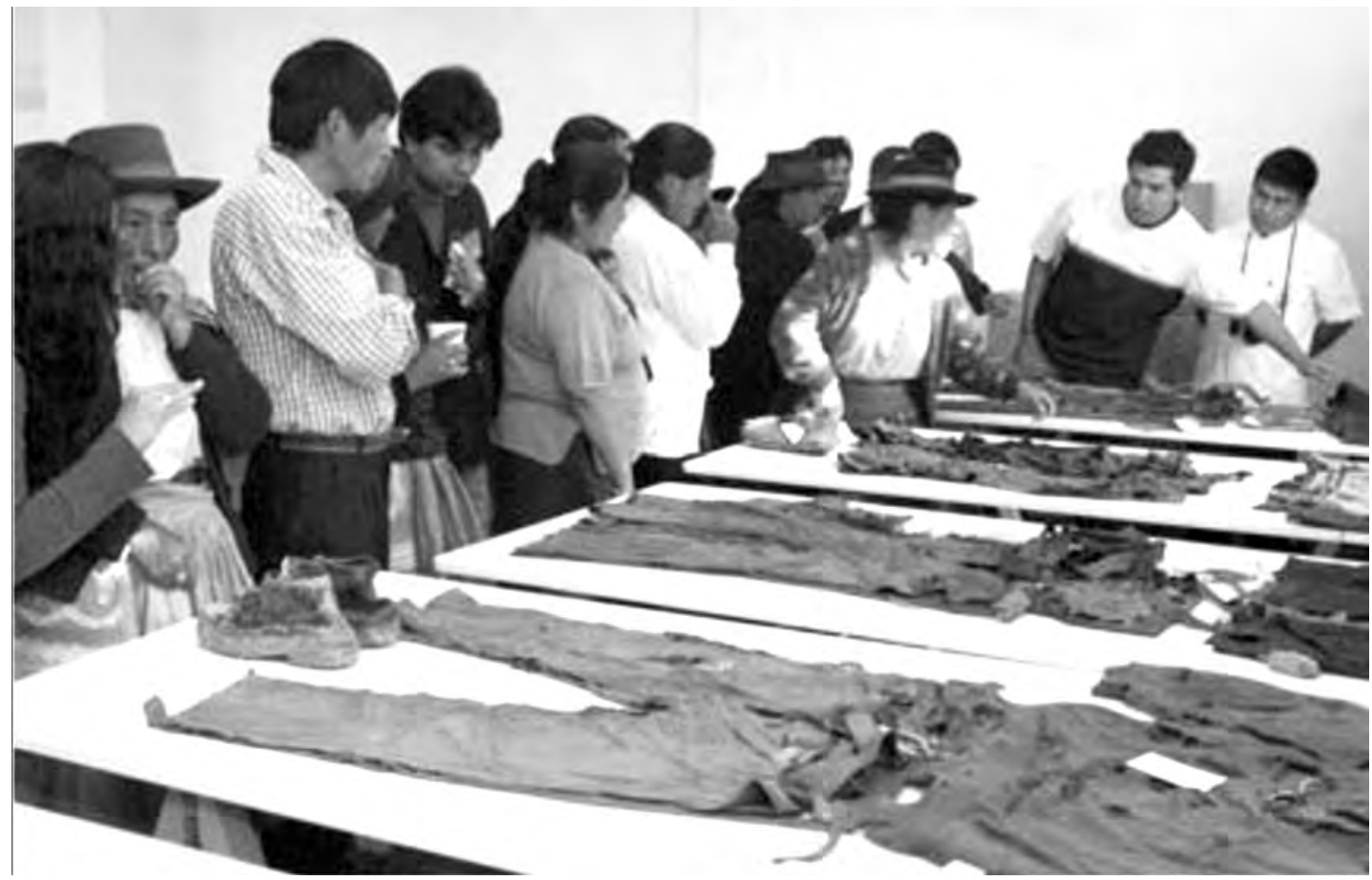

Figura 6. El reconocimiento de las prendas por parte de los familiares es un proceso doloroso y poco eficaz en el proceso de identificación de personas. En la vista diligencia celebrada en Ayacucho en setiembre de 2002.

(Fotografía Archivo CVR). 
demostrado ser de poca utilidad en todos los países donde se ha aplicado, por cuanto la problemática en torno a la desaparición de personas es en extremo compleja.

En casos muy especiales, como por ejemplo el de Guatemala, donde la ropa que utilizan las personas en su mayoría población indígena, quienes fueron los mayores afectados por la violencia, es característica para cada región e incluso para cada persona; la aplicación de este recurso ha tenido resultados positivos 4 .

Lastimosamente no es lo generalizado y aunque eventualmente se pueden presentar situaciones similares, sólo es recomendable acudir al reconocimiento de prendas de vestir como método de individualización. Tanto en el Perú como en otras latitudes, las prendas de vestir suelen tener características comunes entre las diferentes personas. Si bien eventualmente pueden encontrarse prendas características de ciertas comunidades, existen múltiples posibilidades en torno al hallazgo de estas en un contexto forense determinado, entre las que se cuenta de manera no poco frecuente, el préstamo o intercambio de prendas entre víctimas que están siendo desplazadas constantemente e incluso el despojo de la totalidad de sus prendas. Estas prácticas son particularmente evidentes en casos de grandes conflictos o en situaciones de graves violaciones a gran escala, donde los estados tienen una participación tal, que muchas víctimas permanecen por largos períodos en centros de reclusión.

Sin redundar más en los métodos indiciarios se hará énfasis en los dos métodos que llaman la atención en especial de aquellos que desconocen el tema, porque parece a primera vista la manera más económica y rápida de lograr la identificación de víctimas NNs y por ende de personas desaparecidas y porque de manera casi mágica nos devuelve el rostro de los seres que buscamos: la reconstrucción facial y la superposición cráneo-foto.

\section{La reconstrucción facial}

Según Krogman e Iscan (1986) ésta es la técnica más popular para la reconstrucción de rasgos faciales y su uso se remonta a 1881 . Con dos sistemas básicos, la reconstrucción gráfica y la tridimensional, se ha visto popularmente como una técnica útil en la identificación de personas. Sin embargo, después de innumerables estudios, se llegó a la conclusión de que es un método de individualización en ocasiones útil, pero nunca un método eficiente para la identificación fehaciente de víctimas NNs.

La técnica parte del principio de que el tejido blando reproducirá con cierta fidelidad los rasgos faciales dados por el tejido óseo. Sin embargo "muchos detalles del tejido blando no se reflejan directamente en el cráneo" (Ubelaker, 1989), de modo que la reconstrucción facial representa sólo un acercamiento, una aproximación al rostro de un individuo por identificar. Durante el siglo XIX se llevaron a cabo estudios con relación a la variación del grosor del tejido blando por poblaciones y sexos en individuos adultos (técnica no recomendable cuando de individuos infantiles se trata), y a la observación de la variabilidad antropométrica. De acuerdo a Krogman e Iscan (1986), estos estudios llevados a cabo tanto en cadáveres como en individuos vivos por medio del ultrasonido son la base para la técnica tal y como se le utiliza hoy día. Es de suma importancia recalcar que de estas tablas de grosor de tejido blando y antropométricas no hay estudios serios sobre población latinoamericana, ni

\footnotetext{
${ }^{4}$ Se puede precisar que los huipiles, vestimenta tradicional femenina, presenta en su decoración motivos únicos para cada persona. Igualmente sucede con los aretes o las hebillas de los cinturones masculinos, que suelen ser elaborados por miembros de las familias con diseños también específicos, lo cual ha permitido identificar a un porcentaje de las víctimas.
} 
peruana, lo cual conlleva a que la aplicación de los estudios americanos y europeos en población latina tengan implícito un sesgo adicional al que la técnica de por sí conlleva.

La reconstrucción facial consta de al menos tres pasos (Rodríguez, 1994). El primero es la restauración y consolidación de los restos. El segundo paso corresponde a la descripción morfológica y métrica, como condición previa para la reconstrucción. En éste se debe determinar el sexo, y estimar tanto la edad como los ancestros y la constitución física del individuo. Además se requiere realizar observaciones craneométricas y patológicas adicionales, con base en una serie de mediciones de proyección y descriptivas, que darán como resultado la forma de los ojos, previo conocimiento de su tamaño e inclinación, así como la proyección, anchura y forma de la nariz y los pómulos, la anchura del rostro, la forma y proyección del mentón y la relación entre el tejido blando y duro, aspecto que variará dependiendo del tipo de oclusión o del prognatismo maxilar o mandibular.

En este punto es necesario insistir de nuevo en la importancia de que la reconstrucción facial sea el producto de un trabajo interdisciplinario, de manera que se garantice el concurso y la asesoría de médicos, artistas con conocimientos de la figura humana, odontólogos y por supuesto, antropólogos físicos o forenses con la experiencia y la idoneidad suficiente en la materia que incluye la experiencia en este campo. Helmer et. al (1993) sugieren que quien lleve a cabo una reconstrucción facial debe contar con el conocimiento técnico y con la habilidad técnica y escultórica necesaria. Un exceso en la inspiración artística puede llevar a errores y el desconocimiento total de elementos artísticos producirá reconstrucciones poco reales. Condición previa e indispensable es no haber conocido fotos de la víctima, pues esa información viciaría la reconstrucción. Una vez hechas las observaciones métricas, morfológicas y osteopatológicas, algu- nos especialistas recomiendan la elaboración de moldes en yeso del cráneo para evitar daños al mismo durante el proceso. Culminados los pasos anteriores se identifican los puntos craneométricos para establecer con las barras calibradas el grosor del tejido blando. Éste variará según el sexo y el patrón racial en el que se ha ubicado al individuo en los estudios de descripción previos (Krogman, 1986; Ubelaker, 1989; Rodríguez, 1994). Se procede entonces a la reconstrucción facial gráfica (Ubelaker, 1989), mediante la elaboración de perfiles en norma frontal y lateral, con base en fotografías escala 1:1 en plano de Frankfurt. En ellos deberán estar resaltados los puntos craneométricos y se habrá establecido el grosor del tejido, como condiciones previas para proceder a la reconstrucción.

Culminada la reconstrucción gráfica, se procederá a la elaboración de la reconstrucción tridimensional, que requiere de mayores recursos materiales y humanos, por lo que no siempre se recomienda llevarla a cabo, sobre todo porque la reconstrucción gráfica puede ser suficiente para proceder al cotejo o la búsqueda de un individuo.

Una vez ubicadas las barritas calibradas en el molde en yeso y utilizando plastilina o arcilla, según el tiempo y recursos disponibles, se procede a rellenar los músculos faciales, para finalizar colocando la piel. Los ojos pueden ubicarse durante el proceso con moldes en yeso. La forma de la nariz, dependerá de diversos factores. El perfil puede variar, así como la punta y la anchura de la misma; su grado de proyección se basa en los huesos propios y maxilares y se modifica a partir de los cambios que se dan a lo largo de la vida del individuo. En la boca se cuidará de observar rasgos como la anchura y el grosor de los labios. La forma de las cejas, las arrugas o características más específicas, será difíciles de inferir, al igual que la mayor parte de las características de cada rostro dependiendo de la especificidad de cada individuo, según las características de sexo, edad y patologías, entre otros. 

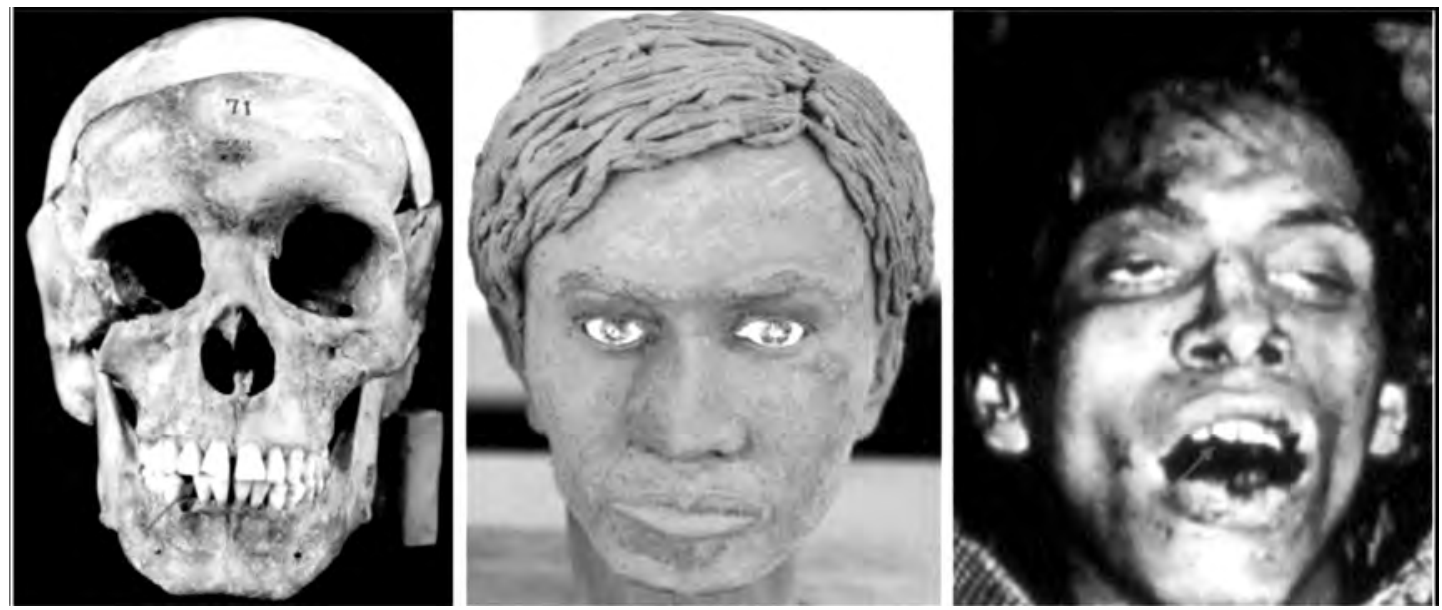

Figura 7. La reconstrucción facial en ocasiones se supone ser orientadora, sin embargo en casos como el representado, sería la fractura del diente (señalada con la flecha) lo que conllevaría a la identificación, obviamente si se conoce la identidad del cadáver en la foto. La reconstrucción facial al centro, tiene pocas similitudes, casi ninguna, con la foto (Foto Laboratorio de Antropología Física de la Universidad Nacional de Colombia).

El gran inconveniente de esta técnica es que la reconstrucción depende en gran medida del criterio de quien elabora la reconstrucción, donde, para estructuras como las orejas por ejemplo, se deben tener en cuenta puntos como la ubicación del tubérculo de Darwin, cuya forma es específica para cada persona y no puede inferirse a partir del cráneo. Situación similar se presenta con el cabello, la barba, su color, longitud y forma, además del color de la piel y los ojos, cuyas características dependen de dicho criterio. Incluso en casos donde algunos de estos elementos se conservan, reconstruirlos es complicado, pues los procesos de descomposición y tafonomía afectan su forma y color de manera significativa. Por estas razones muchos rasgos quedarán inescrutables y solo se logrará una aproximación a la fisonomía real (Neave, en Helmer et al., 1993).

Se debe recordar que la identificación de personas es una de las finalidades de la mayor parte de los estudios forenses (Ubelaker, 1989) y en este proceso, la reconstrucción facial actúa como un elemento de individualización. Aunque en ocasiones se logran resultados sorprendentemente similares, no debe considerarse como un elemento de identificación.
Las investigaciones llevadas a cabo por Helmer et al (1993) con especialistas en la materia de varios países, sobre el uso de este método en procesos de identificación concluyeron que aunque hay consenso en que en gran cantidad de casos más del 50\% de los rasgos coinciden, este porcentaje no es suficiente para establecer una identificación fehaciente, concluyendo que el éxito de este método no es convincente.

Los grados de semejanza que se tomaron para llegar a estas conclusiones, tienen como base una escala de 1 a 5, con grados de observación que van desde una "gran semejanza" hasta una "semejanza nula". El resultado que se logra con la reconstrucción facial es que de un universo determinado de individuos, se reduzca la muestra a unos pocos pasando de allí a aplicar métodos que permitan una aproximación fehaciente a la identidad deseada. Por demás, son innumerables los reportes de casos de reconstrucciones faciales donde el grado de semejanza con las probables víctimas es absoluto, pero el cotejo de historias clínicas u odontológicas preexistentes, dio como resultado la identificación negativa de manera concluyente. Por tanto es una técnica que se sugiere sea utilizada de manera cuidadosa 
teniendo en cuenta que su utilidad real es mínima si se mide el costo beneficio que implica.

\section{La superposición cráneo-foto}

Otros métodos como la superposición cráneo foto, habían demostrado ser aparentemente exitosos (Burns, 1999). Sin embargo a partir de la revisión concienzuda de algunos casos, se encontraron graves errores en la asignación de la identidad a partir de este método. Lo más complejo en torno a la superposición cráneo foto es que se debe garantizar el equipo técnico apropiado así como el personal especializado. Tanto el software como el hardware requeridos son específicos con éste fin y por ende quien lo utiliza debe estar adecuadamente capacitado. Así mismo se requiere que los elementos a cotejar se encuentren en óptimas condiciones de conservación, a lo que se agrega que la calidad, tamaño y orientación de la foto y del cráneo sean coincidentes, siendo necesarios todos estos elementos para lograr la precisión requerida (Krogman e Iscan, 1986).

El método consiste básicamente en tomar una imagen del cráneo, a la cual se le superpone una fotografía o video de la persona que se busca identificar. Para lograrlo se requiere de un equipo de computación y video complejo, nada económico, que cuenta con un software especial que permite la toma de imágenes y su posterior superposición. A lo anterior se agrega que tanto el cráneo como la foto deben tener las mismas proporciones, donde la foto debe ser de tamaño natural, evitando distorsiones; y lo que es más complicado aún se debe lograr exactamente la misma posición entre ambos cuando lo más común es que las fotografías con frecuencia son tomadas en ángulos desviados en cualquier sentido, ya que por obvias razones, y a menos que se trate de una investigación científica, no tienen como fin ser posteriormente comparadas a nivel forense. Según Sánchez Sánchez (2005:209) los proble- mas más frecuentes que se plantean con este tipo de técnica son, entre otros, el tamaño de la cabeza en la fotografía, la dificultad en situar el cráneo en la misma posición que tiene la cabeza en la fotografía, donde los ángulos de rotación y la inclinación varían y es difícil de determinar la posición exacta sin los instrumentos adecuados. Otro factor determinante es el tiempo y los recursos requeridos, en tanto con frecuencia es necesario repetir las fotografías en diferentes posiciones.

A partir de este trabajo de superposición cráneo-foto y hasta la introducción de la computadora, se ha acudido a sistemas que son parecidos a este primero aunque algunos con modificaciones que permiten una mayor comodidad a la hora de superponer como es la proyección del cráneo en una fotografía o acudir a programas no aptos con este fin, que han sido creados con fines audiovisuales diferentes al de la identificación y que generan amplias distorsiones en las imágenes utilizadas, predeterminando los resultados a lo que se quiere obtener.

En casos donde es imposible contar con otros métodos sea por que no existe la información preliminar suficiente, es decir cartas dentales, historias clínicas o por que no se logra acceder a muestras adecuadas para llevar a cabo análisis de ADN, el juicio de identidad quedará en manos de la autoridad quien decidirá si con la cantidad de elementos indiciarios presentados por los peritos, incluyendo la reconstrucción facial, la superposición foto-cráneo, la descripción de las prendas, y la información brindada por los familiares puede establecer una identificación fehaciente y emitir así un juicio de uniprocedencia. Lo anterior, actuando según el criterio propio, con base en los elementos legales que la ley le faculta sin dejar de lado las implicaciones jurídicas, sociales y psico-afectivas que la identificación de un NN implica para todas las partes involucradas. 


\section{Conclusiones}

Es importante entender que la identidad no es un aspecto meramente biológico o social o jurídico, sino una conjunción de todos ellos. Se nace con unas características físicas determinadas, individuales, únicas, pero es la sociedad que legitima esa identidad y es el estado el que la valida jurídicamente. Es un derecho, que es inherente a cada ser humano y como tal debe ser respetado, lo que en consecuencia genera una serie de responsabilidades y deberes de parte de quienes tienen a su cargo la identificación de personas.

En ese sentido, la identificación de personas es básica en nuestra interrelación cotidiana. Desde esta perspectiva y en virtud de la necesidad de ofrecer una serie de garantías y derechos a la sociedad y los individuos, el Estado y la sociedad misma deben garantizar y buscar que ninguna persona quede como víctima NN en situaciones donde la violencia o los desastres naturales pongan en riesgo su identidad y la de su grupo familiar o social, así como su situación psicoafectiva.

Es así como en diferentes lugares del globo se han llevado a cabo varios estudios para lograr identificar de manera fehaciente a los sujetos, primero con base en estudios antropométricos, pasando por el análisis de las huellas, los dientes, la reconstrucción de los rasgos faciales hasta llegar a técnicas más modernas y específicas como el análisis del ADN.

En esta suma de métodos, las huellas dactilares son bastante útiles cuando aún hay tejido blando, incluso momificado; las características dentales, de las historias clínicas cuando hay evidencia de patologías que dejan huella en el hueso, así como el análisis de ADN han contribuido y lo siguen haciendo, también de manera amplia pero sobre todo precisa, cuando hay información previa con la cual comparar.

Es importante que durante el proceso de análisis y recuperación de restos se establezca con precisión que solamente es posible llegar a la identificación fehaciente desde la antropología forense, en la medida que se cuente con los elementos de juicio suficientes y que por tanto, todos aquellos métodos que hacen parte del proceso aportando elementos, indicios, hacen parte de la individualización, de reducir el universo de individuos a identificar, más no lograrán por si solos identificar.

Sin embargo se resalta que la decisión final, el juicio de identidad, estará en manos de la autoridad a cargo del caso, quien sobre la base de la información puesta a su disposición por los peritos, llegará a una conclusión efectiva y eficiente y tomará una resolución con base en ella.

No sobra recordar que la calidad del perito se basa en la experiencia académica y profesional sobre una ciencia, un área o una disciplina determinada y que en esta medida solo quien logre sustentar esta calidad puede interferir en un proceso judicial, en un proceso forense, a solicitud de una autoridad.

Cualquier método de identificación debe ser realizado por un grupo interdisciplinario de peritos antropólogos forenses, médicos, genetistas, odontólogos e incluso artistas que tengan conocimiento en la materia y amplia experiencia.

De acuerdo a las recomendaciones anteriores y considerando que la información que el perito brinda, según su experiencia y capacidades, la autoridad (fiscal o juez) debe concluir en un juicio de uniprocedencia, que permitirá identificar fehacientemente de manera positiva o negativa a una víctima NN. No se debe olvidar el peso legal, social, psicológico y afectivo que este proceso involucra así como las consecuencias que implica, en torno a la manera en que afectará a las familias, a la sociedad y al Estado implicados directa o indirectamente en cada uno de los casos, donde la identidad de las víctimas y la responsabilidad penal de los posibles victimarios debe definirse con base en dicha decisión. 


\section{Agradecimientos}

El presente artículo compila los resultados de experiencias en el campo de la antropología forense. En ese sentido, es el resultado de la constante interacción con profesionales de ésta y otras disciplinas que han nutrido nuestros conocimientos en el área. Sin embargo su realización se la debo a la insistente sugerencia de Carlos Bacigalupo, quién ha enriquecido el artículo de manera constante con aportes y comentarios y ha colaborado permanentemente en la revisión del manuscrito, por lo cual le agradezco profundamente. Las inconsistencias o errores que pueda haber en el texto, son estrictamente responsabilidad de la autora.

\section{Bibliografía}

Burns, K.R.

1999 Forensic anthropology training manual. Prentice-Hall Inc. Upper Saddle River, New Jersey.

Caballero, Hugo

2006 Fundamentos de odontología forense. Manuscrito elaborado para la diplomatura en Antropología Forense y Derechos Humanos, Facultad de Ciencias Sociales, Universidad Nacional Mayor de San Marcos.

Comisión de la Verdad y la Reconciliación (CVR)

2003 Informe Final, Lima.Coordinadora Nacional de Derechos Humanos 2006 Informe Anual 2005. Lima.

Gatliff, B.P.

1984 "Facial Sculpture on the Skull for Identification". En: American Journal of Forensic Medical Pathology 5 (4)327. 332.

Gatliff, B.P., Clyde C. Snow

1979 "From Skull to Visage". En: Journal of Biocommun. 6 (2):27-30
Helmer, R. P.

1987 "Identification of remains of Josef Mengele". En: Journal of Forensic Science: 32 (6): 1622-1644.

Helmer, Richard, S. Rohricht, D, Petersen, and F. Mohr

1993 "Assesment of the reliability of facial reconstruction". En: Forensic Analysis of the Skull: 229-246 Iscan et al. editores. Willey-Liss Inc. Ed.

Krogman, W.M. y M. Iscan

1986 The human skeleton in forensic medicine. Charles Thomas Publish. Springfield. Iscan, Y. I., y Helmer, R.P

1993 Forensic Analysis of the Skull. WileyLiss, New York.

Iten, P.X.

1987 "Identification of skulls by video superimposition”. En Journal of. Forensic Science: 32 (1): 173-188.

Lorente, J.A. y Miguel Lorente.

1995 El ADN y la Identificación en la Investigación Criminal y en la Paternidad Biológica. Ed. Comares, Granada.

Miguel, Ricardo.

s/f. La Identificación de NNs por Métodos Odontológicos. (Ms)

Lubián y Arias, Rafael.

1975 Dactiloscopia. Editorial Reus, Madrid. Neave, Richard

1980 "Facial reconstruction of skeletal remains, 3 egyptian examples". En: MASCA Journal 1(6):175-177, Museum Applied Science Center for Archaeology, University Museum, University of Pennsylvania.

1988 "Facial reconstruction of unidentified King's Cross Fire Victim and the Great Harwood Murder Victim: an Assesment of the Problems and 
Results". En Actas del Simposio Internacional Advances in Skull Identification Via Video Superimposition.

Prag, Jy R. Neave

1997 Making Faces, Using Forensic and Archaeological Evidence. British Museum Press. Londres.

Paredes, Manuel

1998 La prueba del ADN en la investigación del delito y la filiación. Fundamentos de genética forense, $1^{\mathrm{a}}$. arte. Instituto Nacional de Medicina Legal y Ciencias Forenses, Departamento de Docencia y Formación Especializada, Bogotá.

Rhine, J.S.

1984 "Facial Reproduction in Court". En: Human Identification Case Studies in Forensic Anthropology: 357-362, T.A. Rathburn and J. Buikstra (Eds.), III Charles Thomas Publishers. Springfield

Rodríguez, José V.

1994 Introducción a la antropología forense, análisis e interpretación de restos óseos humanos. Anaconda Editores, Bogotá. Sánchez S., José A.

2005 "Aplicaciones del Análisis de Imagen al estudio de la paleopatología y de la historia: Aplicaciones a las investigaciones en curso de Extremadura". Escuela de Medicina Legal, Facultad de Medicina, Universidad Complutense, Madrid En: http://www.dip-badajoz.es /publicaciones/reex/rcex_1_2005/ estudios_10_rcex_1_2005.pdf_(consultado el 09 de marzo de 2007).
Sánchez, Sonia y Mariela Vargas

1993 Identificación Criminal, el ADN como una Nueva Herramienta para la Identificación de Delincuentes. Editorial Jurídica, Bogotá.

Simonin, C.

1982 Medicina Legal Judicial. Edit. Jims, Barcelona

Snow, C.C., Gatliff, B.P., McWilliams K.

1970 "Reconstruction of Facial Features from the Skull: An Evaluation of its Usefulness in Forensic Anthropology". En: American Journal of Physical Anthropology 33:221-228.

Ubelaker, D.

1989 Human Skeletal Remains. Excavation, Analysis, Interpretation. Smithsonian Inst. Taraxacum, Washington.

Valdés, Yesid, Casas, A. Rodríguez, J.V., H. Polanco

1995 Odontología Forense. Ecoe Ediciones. Bogotá.

Velásquez, Luis G.

1990 Apuntes de Criminalística. Universidad Libre de Colombia, Seccional Atlántico, Barranquilla.

Vignal, Jean

1998 Reconstitution Faciale Assistée par Ordinateur. D o $n$ é e s Tomodensitométriques, Deformation Dímage ou "Warping". These de grade pour obtenir le grade de Docteur, specialité Anthropologie. L'Université de Bordeaux I.

Villalaín Blanco, J.D. FJ Pouchalt Fortea

2000 Identificación Antropológica Policial y Forense. Tiran Le Blanch Eds. Valencia. 